\title{
Las bandas juveniles violentas de Madrid: su socialización y aculturación
}

\author{
María Jesús Martín, ${ }^{1}$ José Manuel Martínez ${ }^{1}$ y Alberto Rosa ${ }^{2}$
}

Forma de citar Martín MJ, Martínez JM, Rosa A. Las bandas juveniles violentas de Madrid: su socialización y aculturación. Rev Panam Salud Publica. 2009;26(2):128-36.

RESUMEN Objetivo. El presente artículo analiza el discurso de jóvenes involucrados en grupos violentos o bandas juveniles con el objetivo de profundizar en la interpretación que realizan de los procesos de adoctrinamiento, socialización y aculturación en sus respectivos colectivos. Complementariamente, de acuerdo con el marco teórico de partida (teoría de la socialización primaria y teoría de la asociación diferencial) se aborda la dinámica de influencia social con otros referentes sociales de los jóvenes violentos (pareja, trabajo, familia, etc.).

Método. Análisis cualitativo de 40 entrevistas realizadas a jóvenes pertenecientes a bandas o grupos violentos.

Resultados. En concordancia con la "teoría de la socialización primaria" y la teoría de la "socialización diferencial", se puede postular una sobresocialización del grupo violento y una infrasocialización del resto de agentes socializadores. En relación con la supervisión y el apoyo parental, se identifican tres tipos de familias claramente asociadas al problema de la violencia juvenil. La diversidad o unicidad de identidad social de los jóvenes violentos, así como su autoestima y autoconcepto individuales, conforman un conjunto de procesos de gran relevancia predictiva. Finalmente, un buen indicador de la evolución de estos jóvenes es el apoyo social percibido, absoluto y relativo (distribuido entre los distintos agentes de influencia).

Conclusiones. Se ilustra la necesidad de replantear las bases filosófico-epistemológicas y metodológicas de la investigación sobre esta clase de fenómenos sociales, incorporando elementos centrales del enfoque postmoderno, construccionista y crítico.

Palabras clave Identificación social; violencia; problemas sociales; delincuencia juvenil; conducta del adolescente; investigación cualitativa; España.

España carece de un sistema y de un organismo oficiales que analicen la evolución estadística de la violencia juvenil. No obstante, se han contabilizado 21.076 infracciones penales cometidas por me-

\footnotetext{
Universidad Autónoma de Madrid, Facultad de Psicología, Departamento de Psicología Social y Metodología. La correspondencia debe dirigirse a Universidad Autónoma de Madrid, Facultad de Psicología, Departamento de Psicología Social y Metodología. Ciudad Universitaria Cantoblanco. 28049 Madrid, España. Tel. 0349149752 18. Correo electrónico mariajesus.martin@uam.es

2 Universidad Autónoma de Madrid, Facultad de Psicología, Departamento de Psicología Básica. Ciudad Universitaria Cantoblanco.
}

nores de 18 años en 2006; en 2005 fueron detenidas más de 300 personas vinculadas con bandas juveniles y se identificó a más de 2.000 de sus miembros (1). El informe anual Raxen (2) estima que los grupos neonazis o racistas protagonizan más de 4.000 agresiones al año y sitúa a las víctimas entre inmigrantes, indigentes, homosexuales, prostitutas y los jóvenes de diversas tribus (punkis, hippis, siniestros, etc.). Por otra parte, la extensión desde 2002 de las "maras" latinoamericanas (Latin King, Netas y MS-13) en Madrid y Barcelona, siguiendo el modelo estadounidense, es un hecho ampliamente contrastado (3).

\section{Conductas juveniles y grupales} violentas

En el Informe mundial sobre la violencia y la salud (4), se define la violencia como "el uso intencional de la agresión física o de amenazas de agresión contra uno mismo o contra otra persona, grupo o comunidad, que tenga como consecuencia principal una alta probabilidad de producir lesiones, la muerte, mal desarrollo o privación". Esta definición, demasiado genérica, aconseja caracterizar el tipo de violencia investigada $(5,6)$; por otra parte, al menos en España, muchos actos violentos supuestamente interindi- 
viduales, parecen inspirados por normas y hábitos grupales (7). Por ello, investigaciones españolas $(8,9)$ permiten definir la conducta violenta exogrupal como una agresión física, verbal o simbólica realizada por una o más personas que, en tanto miembros de un grupo, tratan de provocar intencionalmente daño físico o psicológico a una o más personas identificadas como miembros de un grupo distinto.

\section{Aportes de la investigación cualitativa}

En las últimas dos décadas se ha registrado un incremento en las investigaciones cualitativas sobre distintas formas de violencia. La "alternativa cualitativa" descansa en la pretensión de compaginar un análisis ideográfico y procesual con controles de calidad que permitan generar y validar teorías $(10,11)$. Algunos autores identifican las características de la investigación cualitativa que son pertinentes para el estudio de la violencia grupal juvenil: a) la interpretación de experiencias contextualizadas en la cultura y en los agentes de influencia; b) su capacidad para generar un conjunto de significados organizados, una teoría sobre parcelas de la realidad social; c) metodológicamente, un procedimiento de análisis riguroso que permita contrastar los resultados y las conclusiones alcanzadas, y d) la posibilidad de triangular sus resultados con los de las técnicas cuantitativas $(12,13)$.

\section{La violencia grupal juvenil desde la perspectiva de análisis del discurso}

Los estudios cualitativos sobre violencia juvenil no son frecuentes en ciencias sociales, aunque diversos trabajos permiten identificar algunos hallazgos importantes.

Klein, Weerman y Thornberry realizaron un análisis comparativo de estudios cuantitativos y cualitativos sobre gangs estadounidenses y pandillas europeas, y encontraron evidentes diferencias relacionadas con la cultura en la que esos grupos se desarrollan. Así, la violencia estadounidense está más vinculada que la europea con aspectos territoriales y actividades delictivas (por ej. el tráfico de drogas), tiene consecuencias más graves y utiliza con mayor frecuencia las armas de fuego. En ambos casos, sin embargo, la pertenencia a grupos violentos está asociada a la autoestima de sus integrantes (14).

En una investigación sobre las actividades de 35 jóvenes punks violentos, de- sarrollada con entrevistas en profundidad y cuadernos de campo, se concluye que la violencia grupal juvenil refleja la interacción entre factores de la "subcultura grupal callejera" y de "actividades rutinarias" (ritualizadas, automatizadas). Según los autores, la subcultura del grupo serviría de marco interpretativo para identificar a los grupos enemigos, establecer las normas que regulan la interacción con los exogrupos y ritualizar la escalada de violencia (desde miradas hasta violencia física). Las actividades rutinarias juegan un papel importante en la justificación del marco interpretativo generado por la subcultura (15).

Vigil y Yun proponen un modelo de acción y reacción, según el cual la violencia grupal estaría influida por múltiples fuentes de marginación de naturaleza macrohistórica, como el racismo y la represión sociocultural, y macroestructural, como la inmigración y la migración centradas en guetos y zonas de marginación formalmente consolidadas). En este marco, las condiciones ambientales, determinadas por la comunidad o el barrio donde residen, y socioeconómicas determinarían las condiciones socioculturales, tales como el difícil acceso a la educación y marginación de minorías. Por último, los factores socioculturales inducirían el surgimiento de condiciones psicosociales que generan una identidad grupal, la cual una vez constituida invierte el sentido de influencia, dado que su centralidad ayudaría a perpetuar las condiciones socioculturales, ecológicas y socioeconómicas, en especial los guetos y sus actividades delictivas (16).

Otros estudios abordaron aspectos concretos de la violencia grupal juvenil. En un trabajo de Yonas, O'Campo, Burke, y Gielen se vinculan la falta de oportunidades de trabajo en empresas locales, la dificultad de acceso a la vivienda y otras características ambientales, con actividades de tráfico de drogas y con la violencia juvenil (17). Reilly, Muldoon y Byrne, a través de un análisis cualitativo con cuatro grupos focales integrados por 28 jóvenes residentes en áreas pobres de Irlanda del Norte, encontraron dos factores asociados a la violencia juvenil: la desventaja social y la identidad de género masculina, construida sociocultural y grupalmente y concretada en una alta capacidad y motivación para la violencia (18). Zimmerman et al. aplicaron cuestionarios y entrevistas a estudiantes de secundaria e identificaron, entre los factores predisponentes, la falta de autocontrol, la necesidad de aprobación, el acoso o la falta de respeto, la exposición a la violencia mediática y los estilos educativos de los padres (19). Abrams y Aguilar realizaron observación participante durante 16 meses en un correccional de Minnesota, y 12 entrevistas semiestructuradas a varones delincuentes de entre 15 y 17 años de edad, cuyos resultados mostraron la importancia del autoconcepto de estos jóvenes, tanto en su historial delictivo como en su potencial cambio. Sus frecuentes reincidencias al salir del correccional estuvieron influidas por la incapacidad de la familia, del trabajo y de la escuela para fortalecer su autoestima (20).

En España se han realizado investigaciones cualitativas sobre tribus urbanas, algunas de ellas violentas (21), y conducta violenta exogrupal $(8,9)$ cuya síntesis permite identificar cuatro factores interrelacionados: a) socioculturales y económicos, precariedad o insatisfacción laboral, dificultad de emancipación, valores sociales frágiles; b) de identidad social, relaciones entre las identidades grupal, laboral o escolar, familiar y de pareja; c) de identidad personal, actitudes positivas, influencia de las normas del grupo violento, alta autoeficacia (individual y grupal), y d) conductuales, experiencia personal, procesos de aprendizaje mediante modelado o aprendizaje observacional, emociones vinculadas a la violencia, automatismos o rituales.

En consecuencia, todo parece indicar que la investigación cualitativa sobre violencia grupal podría ser singularmente apropiada para abordar su complejidad y evolución. Concretamente, el presente trabajo pretende identificar los principales procesos y factores psicosociales que, en los discursos de los jóvenes entrevistados, se encuentran vinculados a la conducta violenta grupal. Se ha tratado de generar una teoría que permita comprender la influencia de los distintos entornos de socialización y de aculturación de los jóvenes violentos en el desarrollo y modificación de comportamientos violentos contra personas que son identificadas como miembros de un grupo rival, o simplemente distinto.

\section{MATERIALES Y MÉTODOS}

\section{Muestra}

La muestra estuvo formada por 20 varones de 17 a 29 años de edad, y residen- 
tes en la Comunidad Autónoma de Madrid, quienes durante el último año habían agredido en tres o más ocasiones a una o más personas identificadas como miembros de grupos diferentes al propio con intención de producir daño físico (cuadro 1).

\section{Instrumento}

Mediante la técnica de entrevista individual en profundidad semiestructurada, se indagó acerca de cuatro factores fundamentales: a) conducta violenta exogrupal; b) grupo violento; c) otros entornos de socialización, y d) características personales. Cada entrevista se aplicó en dos ocasiones diferentes, separadas por un período medio de 44 días (DE 7 días) (cuadro 2).

El orden de las preguntas de la entrevista siguió la técnica del doble embudo. Primero se hicieron las preguntas generales, consideradas emocionalmente poco reactivas, tales como actividades de ocio, percepción de la sociedad y opinión sobre la situación de los jóvenes. A continuación, en la parte central de la entrevista, se concentraron las preguntas más específicas y conflictivas, relativas a la experiencia personal de los entrevistados con la violencia grupal. Ya en la fase final se formularon preguntas que facilitan la distensión y la generación de un clima positivo que los estimulara a seguir colaborando en el futuro, tales como expectativas y deseos relativos a su vida futura, anécdotas personales y familiares, valoración de la entrevista y permiso para volver a contactarlos.

Las entrevistas fueron realizadas en el período comprendido entre el 9 de mayo de 2007 y el 28 de febrero de 2008.

\section{Procedimiento}

La selección de los jóvenes de la muestra se realizó con la ayuda de asociaciones juveniles y otras organizaciones que trabajan áreas sociales, aficionados fanáticos o ultras de equipos deportivos, y profesionales de la intervención social. Con los tres primeros jóvenes seleccionados, se realizó una aplicación piloto de la entrevista destinada a evaluar su desarrollo y su duración, la comprensión de las preguntas, y la pertinencia de sus contenidos. Como resultado de este análisis, se introdujeron cambios tanto en la formulación como en la ordenación del cuestionario.

CUADRO 1. Características principales de los jóvenes violentos entrevistados, Madrid, España, 2008

\begin{tabular}{|c|c|c|c|c|}
\hline $\begin{array}{c}\text { Grupo } \\
\text { (No. de entrevistados) }\end{array}$ & $\begin{array}{l}\text { Edad } \\
\text { (años) }\end{array}$ & $\begin{array}{l}\text { Adscripción ideológica } \\
\text { o política }\end{array}$ & $\begin{array}{l}\text { Agresiones/mes } \\
\text { (media) }\end{array}$ & $\begin{array}{c}\text { Tiempo en } \\
\text { el grupo } \\
\text { (meses) }\end{array}$ \\
\hline \multirow[t]{4}{*}{ Skinheads neonazis (4) } & 25 & Derecha & $8-10$ & 72 \\
\hline & 22 & Extrema derecha & 8 & 42 \\
\hline & 17 & Extrema derecha & 6 & 16 \\
\hline & 18 & Extrema derecha & $10-12$ & 6 \\
\hline \multirow[t]{2}{*}{ Latin King (2) } & 27 & Sin adscripción & $5-7$ & 84 \\
\hline & 18 & Apolítico & 3 & 120 \\
\hline \multirow[t]{3}{*}{ Skin-red (3) } & 19 & Extrema izquierda & $4-6$ & 16 \\
\hline & 21 & Extrema izquierda & $2-3$ & 12 \\
\hline & 18 & Anarquista & 3 & 8 \\
\hline \multirow[t]{3}{*}{ Sharp (3) } & 21 & Extrema izquierda & $3-5$ & 30 \\
\hline & 18 & Anarquista & $1-2$ & 12 \\
\hline & 19 & Izquierda & $1-2$ & 10 \\
\hline \multirow[t]{2}{*}{ Ñetas (2) } & 21 & Sin adscripción & 2 & 42 \\
\hline & 20 & Apolítico & $<1$ (4-6 anuales) & 12 \\
\hline \multirow[t]{2}{*}{ Ultra Sura (2) } & 25 & Derecha & $1-2$ & 78 \\
\hline & 18 & Apolítico & $2-3$ & 14 \\
\hline Frente atléticob (1) & 22 & Derecha & 2 & 24 \\
\hline \multirow[t]{2}{*}{ Anarquista (2) } & 18 & Anarquista & 2 & 12 \\
\hline & 22 & Anarco-socialista & $<1$ (4-5 anuales) & 24 \\
\hline Okupa (1) & 18 & Apolítico & $1-2$ & 8 \\
\hline
\end{tabular}

a Seguidores del equipo de fútbol Real Madrid.

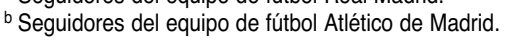

Las entrevistas tuvieron lugar en sitios acordados con los jóvenes participantes. Antes de comenzar las entrevistas, cada participante fue informado acerca de los objetivos de la investigación, de las garantías de anonimato de su identidad y de la confidencialidad de sus declaraciones. Asimismo, se les pidió permiso para grabar sus declaraciones en cintas de audio; cada grabación comenzaba con una descripción por parte del entrevistador, en la cual se confirmaban las condiciones de participación y las garantías mencionadas, y se pedía al entrevistado su aceptación expresa e informada.

La duración media de las primeras entrevistas fue de 91 minutos (DE 19 minutos); en las segundas la duración media fue de 71 minutos (DE 13 minutos). El análisis del discurso se realizó sobre 40 entrevistas, transcritas literalmente en un plazo de 1 a 7 días desde su realización. Las transcripciones siguieron las reglas propuestas por Drew (22).

\section{Análisis de resultados}

Conforme a los objetivos de esta investigación, se realizó un análisis del discurso (23). Mediante esta técnica se pretendió generar una teoría que permitiese comprender el fenómeno estudiado a través de hipótesis concretas, que luego se trataría de refutar mediante su contrastación con nueva información procedente de otras experiencias. El análisis se dividió en dos fases. En la primera entrevista se procuró identificar e interrelacionar los principales procesos y variables potencialmente influyentes en los actos de violencia grupal, con la finalidad de generar una teoría de la socialización de los grupos violentos. Este ejercicio, llevado a cabo con el método de "teoría fundamentada" (23), tenía la finalidad de descubrir variables y procesos, así como sus interrelaciones (13). Para este análisis se partió de una "codificación abierta" coincidente con la presentada en el cuadro 2.

La segunda entrevista se destinó a comprobar o refutar la adecuación de las nuevas declaraciones a la teoría generada en el primer análisis. En tal sentido, se realizó una codificación axial, de naturaleza diacrónica, clasificando las unidades de significado $^{3}$ en tres entornos, que incluyen socialización previa, constitución y evolución del grupo violento (cuadro 3).

Operativamente, se aplicó el método comparativo constante (24), que trata de

\footnotetext{
Párrafos aislados o conjunto de párrafos que muestran una unidad de significado propia, resultado de la interacción lingüístico-simbólica entre entrevistado y entrevistador.
} 


\section{CUADRO 2. Contenidos principales de la entrevista a jóvenes violentos (codificación abierta), Madrid, España, 2008}

\begin{tabular}{|c|c|}
\hline \multicolumn{2}{|l|}{ Episodios de violencia exogrupal } \\
\hline \multicolumn{2}{|c|}{$\begin{array}{l}\text { Antecedentes: lugares de reunión, actividades grupales realizadas, propuesta de agresión, motivos } \\
\text { alegados, emociones expresadas, otros factores }\end{array}$} \\
\hline \multicolumn{2}{|c|}{$\begin{array}{l}\text { Planificación: criterios de elección de la víctima, planificación concreta de la acción, presencia de armas u } \\
\text { otros artefactos, otros factores }\end{array}$} \\
\hline \multicolumn{2}{|c|}{$\begin{array}{l}\text { Rasgos generales del enfrentamiento: entorno, características de los participantes, posibles testigos, otros } \\
\text { factores }\end{array}$} \\
\hline \multicolumn{2}{|c|}{$\begin{array}{l}\text { Características específicas del enfrentamiento: desencadenantes, tipo, duración e intensidad de la } \\
\text { agresión, emociones, comportamiento de testigos, otros factores }\end{array}$} \\
\hline \multicolumn{2}{|c|}{ Finalización del enfrentamiento intergrupal: motivos, desencadenantes, acciones } \\
\hline \multicolumn{2}{|c|}{$\begin{array}{l}\text { Comportamiento individual y grupal posterior: interpretación del episodio violento, satisfacción individual y } \\
\text { grupal, consecuencias, emociones, otros factores }\end{array}$} \\
\hline \multicolumn{2}{|l|}{ El grupo violento } \\
\hline \multicolumn{2}{|c|}{ Características principales: actividades, composición, historia } \\
\hline \multicolumn{2}{|c|}{ Relaciones intragrupales } \\
\hline \multicolumn{2}{|c|}{ Importancia absoluta y diferencial respecto a otros entornos de socialización } \\
\hline \multicolumn{2}{|c|}{ Satisfacción personal en el entorno } \\
\hline \multicolumn{2}{|c|}{ Constitución/inserción en el grupo } \\
\hline \multicolumn{2}{|c|}{ Evolución del grupo: integrantes, actividades, relaciones intra e intergrupales } \\
\hline \multicolumn{2}{|c|}{ Otros entornos de socialización (columna izquierda) y variables analizadas en cada entorno (columna derecha) } \\
\hline La familia & Características principales \\
\hline La pareja & Importancia absoluta y diferencial \\
\hline La institución educativa & Satisfacción personal en el entorno \\
\hline La institución laboral & Relación con violencia exogrupal \\
\hline Otros grupos de pares & Resolución de conflictos \\
\hline \multicolumn{2}{|l|}{ Otras fuentes de socialización } \\
\hline \multicolumn{2}{|l|}{ Rasgos personales } \\
\hline \multicolumn{2}{|l|}{ Valores sociales, ideología } \\
\hline \multicolumn{2}{|l|}{ Autoestima: general y específica } \\
\hline \multicolumn{2}{|l|}{ Autoconcepto: general y específico } \\
\hline \multicolumn{2}{|l|}{ Autoeficacia: general y específica } \\
\hline \multicolumn{2}{|l|}{ Expectativas en la vida } \\
\hline Percepción de la violencia grupal & \\
\hline
\end{tabular}

generar inferencias teóricas provisionales, coherentes con las opiniones expresadas por los jóvenes violentos. Cada nueva declaración sobre un mismo tema se compara con las hipótesis previas para estimar su coherencia teórica. Las discordancias observadas obligaron a reformular las hipótesis para incluir nuevas perspectivas o, en su defecto, clasificar los contenidos, es decir, las distintas percepciones. Como resultado de este proceso se establecieron algunas hipótesis "universales" y otras hipótesis "parciales". Una hipótesis se consideró universal o invariante si cumplía dos condiciones: a) encontró apoyo empírico concreto en las declaraciones de todos los sujetos, y b) no hubo declaración alguna que la invalidara total o parcialmente. Una hipótesis se consideró parcial si incluía diversidad de interpretaciones sobre una cuestión concreta, bastando para ello que existiera una sola declaración incongruente con la hipótesis previamente establecida.

\section{Controles de calidad del análisis del discurso}

La primera fase del análisis del discurso, dedicada a generar teoría, fue desarrollada íntegramente por un miembro del equipo de investigación con amplia experiencia en análisis cualitativo. Los resultados obtenidos y el material original que los fundamenta -declaraciones literales de todos los entrevistados en cada bloque temático o hipótesis formulada- fueron sometidos a un reanálisis individual por parte de otros tres miembros del equipo. Su trabajo tenía como objetivo refutar con apoyo empírico - basado en las declaraciones de los entrevistados- las conclusiones del primer investigador y proponer cambios en las hipótesis invariantes o parciales que propuso. Cada investigador preparó una evaluación donde constaba su acuerdo o desacuerdo, y una o más propuestas alternativas. Estos documentos fueron repartidos entre los cuatro investigadores participantes que analizaron y compararon cada informe personal con los del resto de investigadores, de forma todavía individual. Posteriormente, se aplicó la técnica de "acuerdo interjueces" para reanalizar las discrepancias entre los informes individuales hasta llegar a un acuerdo completo, bien mediante la formulación de hipótesis invariantes o de hipótesis parciales. En este caso también bastaba que un solo juez discrepase con la decisión mayoritaria para que se prefiriese la formulación de hipótesis parciales que recogiesen las distintas interpretaciones.

Una vez finalizada esta fase de análisis, se aplicó el mismo proceso para validar o refutar el modelo teórico. Por último, las conclusiones consensuadas fueron contrastadas con los resultados de las investigaciones precedentes y con la teoría psicosocial pertinente, como puede observarse en el apartado de Discusión y conclusiones. ${ }^{4}$

\section{RESULTADOS}

A continuación se sintetizan los principales resultados hallados, clasificados en nueve aspectos principales. Se presentan en forma de hipótesis que fueron deducidas en las primeras entrevistas, y validadas después con el análisis de la segunda ronda de entrevistas.

\section{Un modelo para comprender la violencia grupal juvenil}

La comprensión de la violencia grupal juvenil presenta un carácter procesual y sistémico. Postulamos una influencia progresivamente convergente de variables macro, meso y microsociales que conducen a algunos jóvenes a sufrir, pero también a disfrutar, de una socialización violenta, en la que su interpretación o construcción individual y social produce una interiorización de la violencia grupal como inevitable, necesaria o justa, y en la que las identidades social y personal de los jóvenes se configuran como procesos centrales para comprender su evolución. Este modelo general se representa en la figura 1 y se sustenta teóricamente en una publicación reciente (25).

\footnotetext{
La estructuración de los discursos en función de las hipótesis generadas y su reconfiguración resultante de la fase de refutación se realizaron con el apoyo del programa informático NUDIST (NonNumerical Unstructured Data Indexing Searching and Theorizing).
} 
CUADRO 3. Contenidos principales de la entrevista a jóvenes violentos (codificación axial o procesual), Madrid, España, 2008

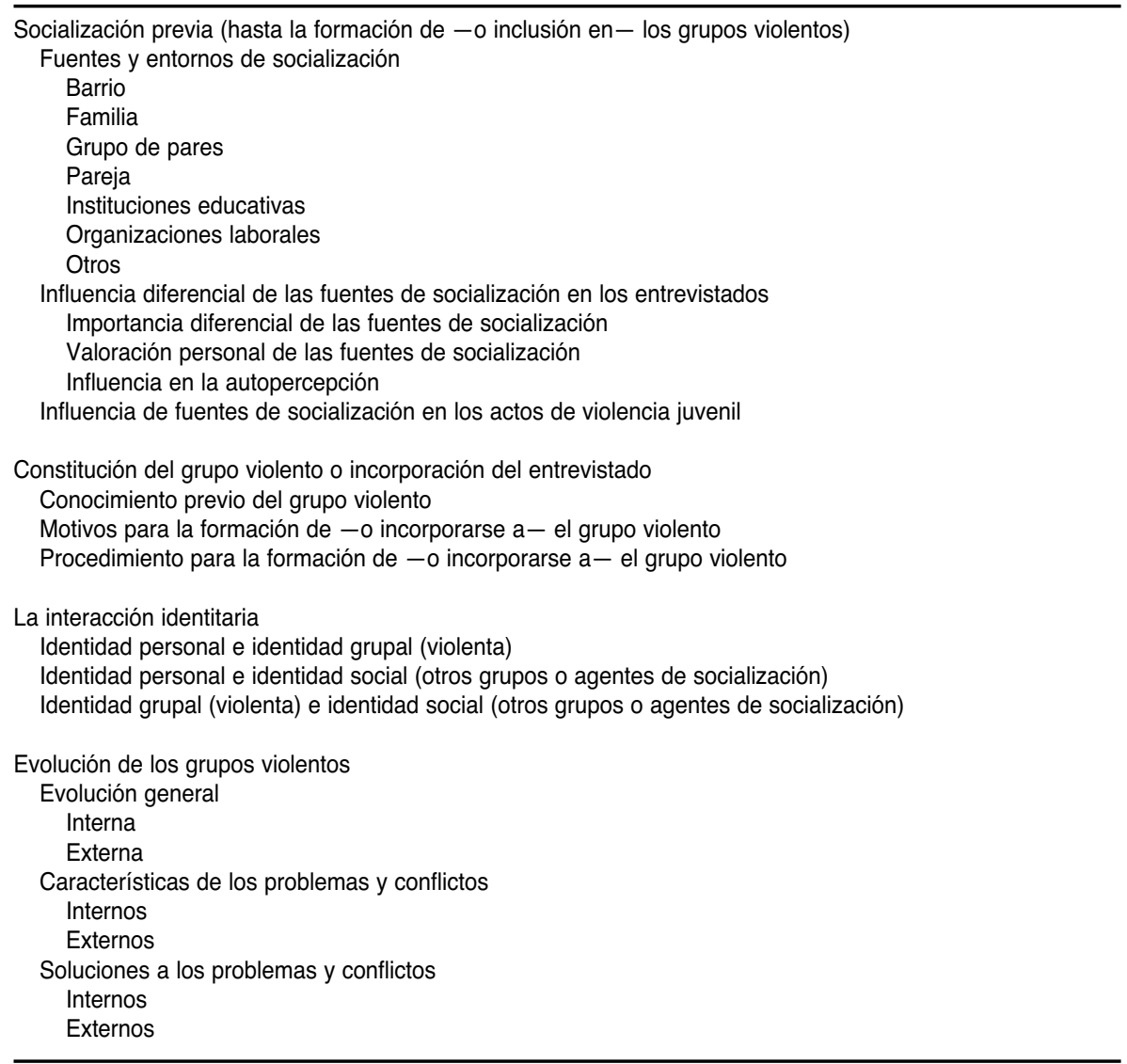

FIGURA 1. Modelo para la violencia grupal juvenil

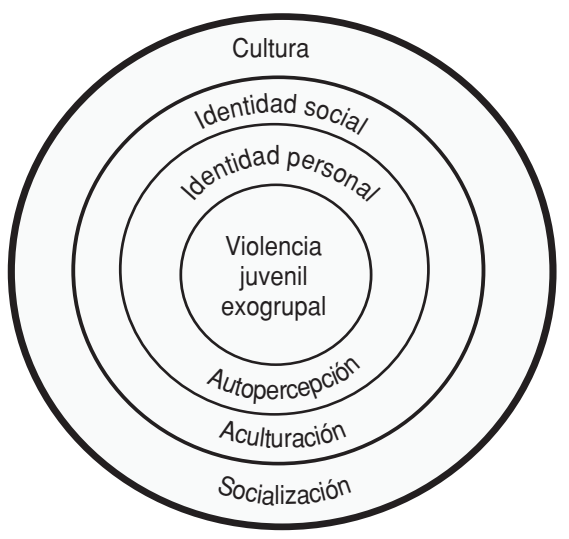

El nivel cultural plantea la influencia diferida y general de procesos de socialización de las diferentes etnias que conviven en un espacio geográfico determinado. Socialización se entiende aquí como la transmisión intergeneracional de normas generales y valores que enmarcan la interpretación de la realidad social que hacen los individuos, grupos, comunidades y organizaciones presentes en un entorno social y geográfico. En el nivel de identidad social se incluyen todos los procesos de influencia que se producen en distintas comunidades, grupos e instituciones a los que pertenecen los jóvenes violentos. En cada uno de ellos se postula un proceso de aculturación singular, que puede describirse de forma general como la influencia que reciben los jóvenes violentos entrevistados por parte de los grupos y organizaciones a los que pertenecen. En estos entornos, la transmisión de normas específicas y actitudes concretas trata de configurar una interpretación de la realidad homogénea entre sus miembros. El nivel de identidad personal está centrado en la autopercepción, es decir, el conjunto de creencias, expectativas y valoraciones relacionadas con el propio individuo entrevistado. Y el nivel central, de carácter más específico, incorpora la experiencia personal sobre la violencia y la interpretación que realiza de sus motivos y efectos. Con estas premisas, se ex- ponen a continuación los principales resultados obtenidos.

\section{Influencia de la experiencia educativa y laboral}

En relación con el sistema educativo, la hipótesis finalmente validada en el análisis del discurso postula que la inadaptación o el fracaso escolar debilitan la autoestima personal y reducen las probabilidades de interiorizar las normas sociales; más aún, sus efectos se prolongan en el tiempo, favoreciendo indirectamente la búsqueda alternativa de fuentes de autoestima social. Concretamente, la inflexibilidad del sistema educativo para satisfacer necesidades motivacionales y emocionales de los alumnos facilita indirectamente que otros agentes de socialización sean más eficaces en la mejora de su identidad personal y social.

En cuanto a la institución laboral, la hipótesis finalmente postulada propone que la realización de un trabajo satisfactorio para el sujeto favorece la normalización de su comportamiento y reduce las probabilidades de caer en conductas violentas. Se considera satisfactorio todo trabajo con retribución económica suficiente y buen clima laboral que incluya apoyo social alternativo. Los trabajos insatisfactorios - mal pagados, precarios o temporales no deseados- provocan en cambio afecto negativo, el cual puede canalizarse (o desplazarse) a través del grupo violento y se concreta en un incremento del deseo de realizar actos violentos.

En este mismo sentido, la inacción o la acción ineficaz en cuanto a la transmisión de normas y comportamientos de otros agentes socializadores, como la familia, la institución laboral o la pareja, favorece la búsqueda activa de opciones que garanticen apoyo social positivo. En el apartado sobre hipótesis y predisposiciones convergentes se comentarán las razones por las cuales esta influencia alternativa recae en el grupo violento.

\section{La influencia de la familia}

La hipótesis fundamental derivada del análisis del discurso en relación con la familia es que existen tres tipos de familia de jóvenes violentos, caracterizadas por patrones diferentes de supervisión y apoyo: anómicas, autoritarias y bipolares.

Las familias anómicas, probablemente las más frecuentes, se distinguen por una 
capacidad limitada de influencia y de coerción, y no mucho esfuerzo por participar en la vida de sus hijos. Tal comportamiento responde a dos razones principales: a) se perciben poco capacitadas o derivan su responsabilidad socializadora a otras instancias, sobre todo a la educativa, o b) se trata de familias que, conscientemente o no, se niegan a reconocer el problema o relativizan su importancia. Los indicios cotidianos de golpes y magulladuras en sus hijos, el ocio de madrugada en preadolescentes, la posesión de navajas, nudilleras de metal u otros artefactos, pueden producir alerta, pero rara vez conducen a un conflicto manifiesto con sus hijos.

Las familias autoritarias se caracterizan por un anhelo abrumador de control, por la explicitación de una serie de normas que regulen el comportamiento de sus hijos y por una permanente presión por inculcarles sus motivaciones y aficiones, dificultando el desarrollo de una autopercepción positiva y diferenciada.

El caso de la familia bipolar es uno de los resultados principales de la codificación axial realizada con los discursos de los jóvenes, la cual ha permitido descubrir familias que a medio o largo plazo modifican de manera extrema sus pautas educativas y socializadoras. En general se trata de familias que al principio son más bien anómicas, pero que cuando deben afrontar consecuencias fuertemente negativas de la conducta de sus hijos, por ejemplo ante denuncia policial o heridas que requieren hospitalización o atención médica de urgencia, reaccionan abruptamente como una familia autoritaria, con castigos, restricciones del ocio y amenazas. El relativo fracaso de sus intentos a corto o medio plazo o los conflictos internos entre ambos padres suscitados por la falta de acuerdo en el modo de encarar el problema, los induce a retomar la estrategia inicial (negación o subestimación del problema) que perdura hasta la siguiente crisis, si es que se produce.

\section{¿Por qué los jóvenes buscan apoyo social alternativo en los grupos violentos? La hipótesis de predisposiciones y habilidades convergentes}

En todos los casos consultados se halló que una parte sustancial de la autoestima desarrollada previamente al ingreso en un grupo se basaba en cierto grado de pre- disposición a la violencia, en experiencias positivas de enfrentamientos interindividuales o intergrupales anteriores, o en habilidades e inclinación hacia los deportes violentos, como las artes marciales o ciertos deportes de contacto.

Con estos resultados, es coherente postular que el hecho de que esta socialización alternativa recaiga en grupos violentos parece deberse a que, previo a su ingreso a dichos grupos, los jóvenes ya poseían predisposiciones hacia la violencia vinculadas a su autoestima personal (se consideraron fuertes, valientes y capaces de defenderse).

\section{Identidad social de los jóvenes violentos}

La primera hipótesis validada en este sentido es que la pertenencia a un grupo violento contribuye fuertemente a definir la identidad social en sus jóvenes integrantes. A la vez, su formulación y validación se basaron en la aceptación de otra hipótesis, de naturaleza descriptiva, que resultó clave para continuar el análisis: es necesario distinguir entre identidad social general (sentido de pertenencia a distintos grupos sociales) $y$ emergente - resultado de experiencias, hábitos, normas o actitudes relacionadas con un grupo determinado en una situación concreta.

Bajo estas premisas, y de acuerdo con los resultados alcanzados, se puede postular que entre los jóvenes violentos parece existir un continuo de complejidad identitaria, que tendría como valor-suelo la "identidad social única" y que variaría en función de la pertenencia y la relevancia de distintos grupos sociales.

También se ha podido establecer que los jóvenes entrevistados difieren en el grado en que perciben que su conducta violenta provoca un conflicto entre distintos entornos afectivos, como por ejemplo entre grupos de pares y familia.

El cruce de las declaraciones de todos los entrevistados sobre estas dos relaciones conceptuales permitió establecer y validar la hipótesis de que la violencia grupal ejercida está parcialmente influida por la situación de cada joven violento en este continuo y, eventualmente, el grado de conflicto que exista entre las distintas identidades sociales en relación con los actos de violencia grupal.

Más concretamente, la identidad social única incluye una concentración de actitudes, conductas, normas, expectativas y planes de acción en un solo agente de socialización. Esta clase de grupo perisectario promueve la obediencia y la interiorización de las normas grupales a través de rituales iniciáticos de gravedad progresiva, que trasladan a quienes los realizan a dos nuevas experiencias con una fuerte influencia posterior: el afrontamiento de un delito (sea agresión física o robo) y las consecuencias marginadoras resultantes, y la aceptación en el grupo como un miembro de pleno derecho.

El resto de grupos no exige una atención permanente, las normas son menos explícitas y no existen rituales iniciáticos estandarizados ni de graves consecuencias. Sus miembros dirigen la violencia hacia grupos concretos, y mantienen en paralelo relaciones sociales normalizadas en otros ámbitos. La influencia de estos otros grupos sociales o de nuevos agentes de influencia (pareja, trabajo) resulta fundamental para normalizar la conducta, ya que estos jóvenes no suelen abandonar el grupo violento si no hay alternativas de apoyo.

\section{Identidad de género, identidad social y conducta violenta}

En relación con la identidad de género masculina, podemos afirmar que las pandillas o grupos violentos tratan de promover en sus nuevos miembros una identidad masculina y positiva ligada a dos tipos de gestalt interpretativa: a) en grupos de ultraderecha o sin ideología: fortaleza física y psicológica y apoyo incondicional a los miembros del grupo; b) en grupos de otras ideologías: fortaleza psicológica dirigida a la defensa proactiva y reactiva del grupo y de los colectivos minoritarios, sin que se perciba como imprescindible el apoyo incondicional a los miembros del grupo.

Por otra parte, en relación con otras formas de identidad social, se postula que la identidad de género masculina es coherente y armónica con la identidad grupal, está integrada en ella y no presenta conflicto alguno con el resto de identidades (grupal, familiar, laboral).

Los valores tradicionalmente asociados a la masculinidad en las dos versiones mencionadas no entran en conflicto con los valores de otros entornos de socialización de los jóvenes; de hecho han sido potenciados por la familia antes de la pertenencia al grupo violento y parecen ser bien aceptados por el entorno laboral y el escolar. 


\section{Construcción y distorsión de la realidad}

En todos los casos se han podido identificar uno o varios tipos de sesgos o distorsiones sistemáticas en la interpretación que realizan los jóvenes violentos de su realidad, vinculados a su pertenencia al grupo violento o a la relación de este entorno con otros colectivos y personas. Más concretamente, en relación con la violencia ejercida por los entrevistados y por sus respectivos grupos, es muy frecuente observar a nivel individual el estilo atribucional egótico por el que jóvenes de grupos violentos consideran que cualquier acción indebida o injusta fue realizada por causas externas (provocación, agresión simbólica previa, etc.), inestables (circunscritas a esta situación) y específicas (sólo aplicables o esperables en conflictos intergrupales). Fuertemente vinculado al sesgo anterior, pero a nivel grupal, el error de atribución último o sesgo fundamental intergrupal se emplea para culpabilizar de la violencia a los grupos rivales o simplemente distintos (en el caso de grupos violentos no extremos) y legitimar la violencia ejercida por él mismo y por su grupo. Operativamente, tal comportamiento favorece la cohesión del grupo e incrementa la autoestima individual y social al considerar que las conductas positivas protagonizadas por miembros del endogrupo se deben a causas internas y estables, mientras que las protagonizadas por personas del exogrupo se consideran fruto de condiciones externas e inestables.

Por tanto, se puede postular que la interacción entre experiencia personal, identidades grupales y condiciones socioculturales facilita las decisiones conductuales a través de un procesamiento racional o de un procesamiento intuitivo, emotivo-motivacional.

Es muy probable que los grupos violentos promuevan activamente una aculturación explícita e implícita en normas y hábitos agresivos. Al parecer, los jóvenes subestiman la influencia de mecanismos de defensa no conscientes, tanto personales como grupales, que promueven la cohesión grupal y favorecen un sentimiento de pertenencia. Así, por ejemplo, los éxitos en el combate con otros grupos violentos se convierten prontamente en narraciones míticas, probablemente magnificadas, que ayudan a transmitir implícitamente normas de apoyo, de responsabilidad grupal y de reciprocidad. Sobre todo en grupos con alto nivel de experiencia y un gran nivel de control, el papel de los hábitos y de los guiones de conducta que se concretan sobre todo en los "rituales de caza" es muy significativo.

Por otra parte, los resultados apoyan la hipótesis de que la influencia de la violencia ejercida debe ser interpretada teniendo en cuenta los distintos ámbitos en que dicha influencia tiene lugar.

En cada uno de ellos (familia, grupo violento, otros grupos, pareja, trabajo), los significados y su manejo son en gran medida peculiares. Así, por ejemplo, "salir de caza" implica un ritual que es interpretado por todos los entrevistados como un eficaz sistema de protección, de ataque preventivo, que genera seguridad en el endogrupo y temor en el exogrupo; además, lleva aparejados relatos y mitos sobre anteriores agresiones y sus resultados (casi siempre positivos). Fuera de este ámbito, esta gestalt interpretativa carece de sentido, e incluso es frecuente que cuando se aborda el tema con la familia o la pareja, el discurso cambia sustancialmente, se incluyen matices y se suprimen elementos dramáticos. Es probable que los jóvenes no perciban contradicción alguna entre ambos discursos.

\section{Vulnerabilidad e invulnerabilidad grupal e individual}

Desde una perspectiva diacrónica (codificación axial) se ha podido constatar (en la primera entrevista) y validar (en la segunda) que, a excepción de los que tienen una "identidad social única", los jóvenes violentos entrevistados en general experimentan cambios en su percepción de vulnerabilidad grupal y que tal percepción está vinculada también con la autoestima individual.

En 16 de los 20 jóvenes entrevistados se pudo constatar que determinadas condiciones inducían sensación de vulnerabilidad grupal o provocaban conflictos intergrupales o incrementaban la inseguridad personal o aumentaban el deseo de abandonar el grupo violento.

Las principales condiciones que incrementan la sensación de vulnerabilidad son haber recibido una fuerte paliza en un enfrentamiento intergrupal; creer que su familia puede verse afectada directamente por la violencia grupal; padecer una acción directa y sostenida de la familia contra sus comportamientos violentos (especialmente por parte de la madre); haber sido objeto -él o un par de su entorno íntimo dentro del grupo- de detenciones o denuncias policiales; provocar entre los rivales heridos o muertos que creen alarma social en los medios de comunicación; el abandono del grupo por parte de amigos cercanos, y la aparición de nuevos agentes de socialización contrarios a la violencia grupal y que mejoran la autoestima (pareja, un buen trabajo, amigos de la infancia).

\section{Intervención social con grupos juveniles violentos}

A partir de los resultados alcanzados, queda claro que un objetivo fundamental para hacer frente a este problema social es promover y facilitar identidades personales y sociales positivas mediante el estímulo de conductas prosociales, y asegurarse, con técnicas participativas e implicativas, de que estos cambios sean interiorizados. Si se ha de conseguir este objetivo, será necesario complementar acciones individuales con propuestas alternativas socializadoras (ocio, trabajo, amigos, familia), motivantes y satisfactorias, aprovechando los períodos de vulnerabilidad que se suceden naturalmente en los grupos violentos debido a consecuencias negativas de sus acciones o a la influencia de otros agentes socializadores.

\section{DISCUSIÓN}

Los resultados obtenidos son coherentes con la "teoría de la socialización primaria" (26) y con la teoría de la "socialización diferencial" (27). Es posible postular una sobresocialización del grupo violento y una infrasocialización del resto de agentes socializadores. Este desequilibrio se observa nítidamente al analizar la interacción entre identidad personal, grupal y social. Así, cuando la identidad grupal (violenta) es dominante y emergente en diversos ambientes, los jóvenes presentan conductas más extremas y su vinculación con el grupo violento es mayor. En estos casos, la influencia de la familia, de la pareja y de otros agentes suele circunscribirse a sus ámbitos de actuación cotidianos. Esto parece suceder porque los jóvenes violentos ocultan su actuación al resto de agentes socializadores y porque estos no advierten o, en la mayoría de los casos, no quieren advertir estas conductas $\mathrm{o}$, en casos aislados, directamente las aprueban, por ejemplo cuando se trata de hermanos que también pertenecen a esta clase de grupos. 
Estas tendencias de acción parecen estar potenciadas también por una socialización diferencial de género que ha promovido una identidad de género coherente con la identidad grupal y basada en expectativas y habilidades violentas, emergentes e intensas $(18,27)$.

En relación con la supervisión y apoyo parental, los resultados obtenidos son similares a los hallados en dos trabajos sobre prácticas de riesgo en España (8, 28). En ambos se identifican las familias anómicas (negligente o permisiva según los mencionados trabajos) y las familias autocráticas (o autoritarias). Sin embargo, se observa una nueva clase de familia, denominada "bipolar", que supone a medio y largo plazo una combinación de las anteriores. El principal efecto de estos tipos de familias parece ser dificultar el desarrollo de una identidad personal y social positivas e inducir indirectamente a que los jóvenes la adquieran mediante la pertenencia a grupos sociales alternativos (en este caso violentos, elegidos por sus predisposiciones) (8).

La diversidad o unicidad de identidad social de los jóvenes violentos, y su vinculación con la autoestima y el autoconcepto individuales, se configura como un conjunto de procesos vinculados y de gran fuerza predictiva. Resulta especialmente preocupante el caso de los jóvenes de "identidad social única", cuya conducta puede asimilarse en buena medida a la que presentan las sectas con intensos procesos de aculturación de sus miembros, mediante la aceptación pública de normas y la participación en severos rituales iniciáticos (29).

Bajo estas premisas, un buen indicador de la evolución de estos jóvenes es el apoyo social percibido, absoluto y relativo, distribuido entre los distintos agentes de influencia. Los jóvenes insertos en grupos violentos concentran su apoyo social y desarrollan sus actividades y expectativas en este colectivo. Otras dos condiciones favorecen esta fuerte vinculación: la escasez de apoyo social de otros agentes de socialización y la ausencia de conflicto con otros entornos (familia, pareja, trabajo) por motivo de la violencia ejercida.

Finalmente, los resultados empíricos alcanzados en esta investigación son pertinentes para un corolario filosóficoepistemológico que, habiéndose convertido en clásico, es relevante por sus notables consecuencias en la generación y validación de conocimiento en el ámbito de las ciencias.

El debate epistemológico entre positivismo o neopositivismo y marco crítico o posmoderno es un elemento central en la configuración de las ciencias naturales y sociales contemporáneas y está íntimamente relacionado con la elección teórica y metodológica del investigador (30), y su articulación tiene efectos importantes en el estudio de los comportamientos delictivos y antisociales. Así, por ejemplo, resulta esencial decidir si el investigador considera posible la deducción de leyes, principios o teorías sobre la conducta violenta grupal que tengan un valor universal o, por el contrario, si las conclusiones se encuentran limitadas a una cultura, comunidad o grupo concretos. También es muy importante afrontar la polémica sobre la validez temporal de las hipótesis y teorías, dependientes o independientes, de la naturaleza histórica y evolutiva de los procesos, de las comunidades y de las culturas. No obstante, en la actualidad es posible que en buena parte de las ciencias sociales, se encuentre instalada, de forma más o menos explícita, una orientación epistemológica ecléctica que, por ejemplo, considera im- probable al menos a corto plazo el establecimiento y la falsación de leyes psicosociales universales. A partir de estas premisas, el presente trabajo postula que la comprensión de la violencia grupal debe contemplar el enmarque y moldeamiento de la cultura (31); los procesos de construcción social que realizan sociedades, comunidades y grupos (32), y la dualidad interactiva de procesos explícitos e implícitos (33) o conscientes e inconscientes (34-36). Otra consecuencia sustancial del debate epistemológico sobre el estudio de los comportamientos sociales antinormativos se refiere a la influencia de procesos culturales, de las tradiciones de los grupos de investigación, y de los sesgos y heurísticos individuales y grupales que también afectan a los investigadores en la generación y validación del conocimiento científico (37).

Finalmente se puede concluir que, desde la perspectiva epistemológica, el conocimiento teórico y aplicado sobre la violencia grupal debe partir de la comprensión de cómo las personas y los colectivos sociales interpretan y configuran la realidad y cómo estas percepciones influyen en la producción y evolución de los comportamientos delictivos o antinormativos. Más importante todavía, existen fuertes razones para que este objetivo pueda resultar más probable desde una perspectiva filosóficoepistemológica, que combine los paradigmas positivista y crítico, y articule la conveniencia - o la necesidad- de afrontar los problemas o desafíos (sociales y naturales) desde las aportaciones singulares de diferentes disciplinas, con variedad de enfoques teóricos y metodológicos, pero obligadas a generar códigos compartidos y aproximaciones complejas a la realidad.

\section{REFERENCIAS}

1. Ministerio de Interior español. Balance sobre criminalidad y delincuencia de 2006. Disponible en http:/ / www.copslocales.com/balancesobre-criminalidad-y-delincuencia-de-2006vt1465.html, 2007.

2. Informe Anual Raxen. El mapa del odio en España. Madrid: Movimiento contra la Intolerancia; 2007.

3. Iglesias $\mathrm{S}$. Consideraciones de política criminal. Globalización, violencia juvenil y actuación de los poderes públicos. Madrid: Dikinson; 2006.

4. Krug EG, Dahlberg L, Mercy JA, Zwi AB, Lozano R. Informe mundial sobre la violencia y la salud. Washington, D.C.: Organización Panamericana de la Salud; 2003.

5. Chavez V. Operational definitions of youth violence in empirical research. Int Q Community Health Educ. 1999;18(2):237-61.

6. Moser CO. Urban Violence and Insecurity: An Introductory Roadmap. Environment and urbanization. 2004;16:3-16.

7. Martín A, Martínez JM, López J, Martín MJ, Martín JM. Comportamientos de riesgo: violencia, prácticas sexuales de riesgo y consumo de drogas ilegales en la juventud. Madrid: Entinema; 1998.
8. Martín MJ. Violencia juvenil exogrupal: hacia la construcción de un modelo causal. Madrid: Ministerio de Educación y Ciencia/CIDE; 2005.

9. Scandroglio B, López JS, Martínez JM, Martín MJ, San José MC, Martín A. La conducta violenta en grupos juveniles. Características descriptivas. Revista de Estudios de Juventud. 2003;62(3):151-8.

10. Flick U. Introducción a la investigación cualitativa. Madrid: Morata; 2004.

11. Potter J, Wetherell M. Discourse and Social Psychology. London: Sage; 1987. 
12. González Rey FL. Investigación cualitativa en Psicología. Rumbos y desafíos. México DF: International Thomson Editores; 2000.

13. Íñiguez L. Análisis del discurso. Manual para las ciencias sociales. Nueva edición revisada y ampliada. Barcelona: UOC; 2006.

14. Klein MW, Weerman FM, Thornberry TP. Street Gang Violence in Europe. European Journal of Criminology. 2006;3(4):413-37.

15. Kennedy LW, Baron SW. Routine Activities and a Subculture of Violence: A Study of Violence on the Street. Journal of Research in Crime and Delinquency. 1993;30:88-112.

16. Vigil JD, Yun SC. A cross-cultural framework to understand gangs: multiple marginality and Los Angeles. En: Huff CR, Gangs in America III. Thousand Oaks, Ca: Sage Publications, 2002. Pp. 161-74.

17. Yonas M, O'Campo P, Burke JG, Gielen AC. Neighborhood-Level Factors and Youth Violence: Giving Voice to the Perceptions of Prominent Neighborhood Individuals. Health Educ Behav. 2007;34:669

18. Reilly J, Muldoon OT, Byrne C. Young Men as Victims and Perpetrators of Violence in Northern Ireland: A Qualitative Analysis. J Soc Issues. 2004;60(3):469-84.

19. Zimmerman MA, Morrel-Samuels S, Wong N, Tarver D, Rabiah D, White S. Guns, Gangs, and Gossip: An Analysis of Student Essays on Youth Violence. J Early Adolesc. 2004;24(4): 385-411.

20. Abrams L, Aguilar JP. Negative Trends, Possible Selves, Behavior Change: A Qualitative
Study of Juvenile Offenders in Residential Treatment. Qualitative Social Work. 2005;4: 175-96.

21. Feixa C. De jóvenes, bandas y tribus. Barcelona: Ariel; 1998.

22. Drew P. Conversation Análisis. En: Smith JA, Harré H, Langenhove LV eds. Rethinking Methods in Psychology. London: Sage; 1995.

23. Glaser B, Strauss A. The discovery of grounded theory. Strategies for qualitative research. Chicago; 1967.

24. Strauss A, Corbin J. Basics of qualitative research: Grounded theory procedures and techniques. London: Sage; 1990.

25. Martín MJ, Martínez JM, Martín MA. Psicología Social Contemporánea e Intervención Social. Modelo sistémico para orientar la investigación aplicada y la intervención social. Boletín de Psicología. 2007;91:55-82.

26. Oetting ER, Donnermeyer JF. Primary socialization theory: The etiology of drug use and deviance. Subst Use Misuse. 1998;33(4): 995-1026.

27. Howell JC. Preventing \& Reducing Juvenile Delinquency: A Comprehensive Framework. California: Sage; 2003.

28. Musitu G, Molpeceres MA. Estilos de socialización, familismo y valores. Infancia y Sociedad. 1992;16:101

29. Rodríguez P. Adicción a sectas. Barcelona: Ediciones B; 2000.

30. Chalmers AF. ¿Qué es esa cosa llamada ciencia?. Paracuellos del Jarama (Madrid): Siglo XXI de España Editores; 2004.
31. Páez D, González JL. Culture and social psychology. Psicothema. 2000;12(1 Supl):6-15.

32. Gergen KJ. Construir la realidad. El futuro de la psicoterapia. Barcelona: Paidós; 2006.

33. Kruglanski AW. Motivated social cognition: Principles of the interface. En: Higgins ET, Kruglanski A, eds. Social Psychology: Handbook of basic principles. New York: Wiley; 1996. Pp. 493-520.

34. Bargh JA. Social Psychology and the Unconscious. The Automaticity of Higher Mental Processes. Philadelphia: Psychology Press; 2007.

35. Billig M. Discursive approaches to studying conscious and unconscious thoughts. En: Tolman DL, Brydon-Miller M, eds. From Subjects to Subjectivities: A Handbook of Interpretive and Participatory Methods. New York: University Press; 2001

36. Moscovici S, Mugny G, Pérez JA. Les effects pervers du déni (par la majorité) des opinions d'une minorité. Bulletin de Psychologie. 1984: $365-80$.

37. Íñiguez L, Pallí C. La Psicología Social de la Ciencia: Revisión y discusión de una nueva área de investigación. Anales de Psicología. 2002;18(1):13-43.

Manuscrito recibido el 17 de abril de 2008. Aceptado para publicación, tras revisión, el 16 de enero de 2009.

ABSTRACT Objectives. This study explores the subject of youth involved in violent groups or gangs, with the goal of further understanding the indoctrination, socialization, and culturalization processes undergone by youth involved in group violence or gangs. Furthermore, to examine the dynamics between peer pressure and other social factors (dating relationships, work, family, etc.) within the theoretical framework of the theories of primary socialization and differential socialization.

Methods. A qualitative analysis of 40 interviews of youth belonging to violent gangs/groups.

Results. According to the theories of primary socialization and differential socialization, over socialization by the violent group and under socialization by all other social entities can be assumed. Regarding parental supervision and support, three family types were clearly associated with the problem of youth violence. The distinct or unified social identity of the violent youth, as well as their individual self esteem and self image, formed a combination of processes whose relevance was highly predictive. Lastly, an accurate indicator of how these youth mature is their support networkperceived, absolute, and relative (distributed among the various influencing forces). Conclusions. The study clearly outlines the need for re-imposing fundamental philosophical epistemology and methodologies on social forces of this kind, incorporating elements key to the postmodern, constructionist, and opposing perspectives.

Key words Social identification; violence; social problems; juvenile delinquency; social dominance; adolescent behavior; qualitative research; Spain. 